\title{
Command and Control Systems Overall Training Evaluation Software Design and Implementation
}

\author{
Hao Zhang, Yan-Hui Wang \\ Dalian Naval Academy \\ Dalian, China
}

\author{
Shen-Chang Zhang \\ PLA NAVY, No.91278 Army Force \\ Dalian, China
}

\author{
Ting Liu \\ The Second Artillery Engineering University, Shanxi Xi'an 710025 \\ Dalian, China \\ Email: xieliaoxa@163.com
}

\begin{abstract}
In order to improve the automatic and efficient evaluation of Command and Control Systems Overall Training Evaluation, a command and control systems overall training evaluation software has been proposed in this paper. A list of qualitative and quantitative evaluation methods is combined to determine the evaluation index, and VB, MS SQL Server and other technologies are implemented in the design and development of the software. The proposed software is closely attached with the overall training evaluation requirements. Making full use of evaluation data, the speed and efficiency of the evaluation process have been enhanced. The software has its strong practical significance for improving the automation level and scientific evaluation.
\end{abstract}

Keywords-Efficient Evaluation; Command and Control System; Overall trining; Evalustion Software.

\section{INTRODUCTION}

With the comprehensive information construction of the armed forces, the command and control system has its significance no matter in the performance or in the complexity. In actual wars, the requirements of the commander's operation ability have totally changed. [1]But in the training assessment method of command and control system, what applies is still the manually recorded examination data, which requires computer-based statistical analysis with human input. In such way of processing the training assessment, it requires lots huge efforts of manpower, repetitive labour and longer work time, which all limit the efficiency of training evaluation.

Aiming at solving the existing problems in the traditional examination method, we have designed a "Overall Training Assessment Software for Command and Control System". [2]This software mainly serves the command and control system personnel in the battle stations. This software is able to provide superior authority senior officers and the operation department an easy, quick, scientific, and accurate training performance of the assessed personnel, by making a detailed assessment and producing an assessment report. In the software design process, the attention is on the basic functionalities and humancomputer interactive characteristics; the AHP thought and expert quantitative thinking are also taken into account for improving the check method of the examination assessment index, which lead to a more scientific and sound result, and minimize the impact of subjective factors as much as possible. [3]

\section{WHOLE SOFTWARE DESIGN OF THE OVERALL TRAINING ASSESSMENT}

\section{A. The Architecture Design of Software and Network}

In the implementation process, an in-depth research is conducted on relevant operating principles of the command and control system, the assessment standard of the command and control examination, etc. then, regarding to the system's status quo, a detailed design of the system's interface is proposed. [4] This software is developed under Microsoft Windows XP, and adopted Microsoft's Visual Basic 6.0 Chinese business version as the development tool and Microsoft SQL Server 2005 database system in the daemon. The adoption of mature software development tools as well as database system reduces the difficulty in software implementation, and improves the speed of data processing. [5]Following the multidimensional structure model and the modular structure of design and development, both the maintainability and stability of the system have been ensured.

The command and control system's overall training assessment software requires the connection of the Ethernet and database server, and is an implementation of the $\mathrm{C} / \mathrm{S}$ structure, where not only the tasks are reasonably distributed to the client and server, but also the functions vary according to the user's access information. The development environment and network structure is shown in Figure 1. 


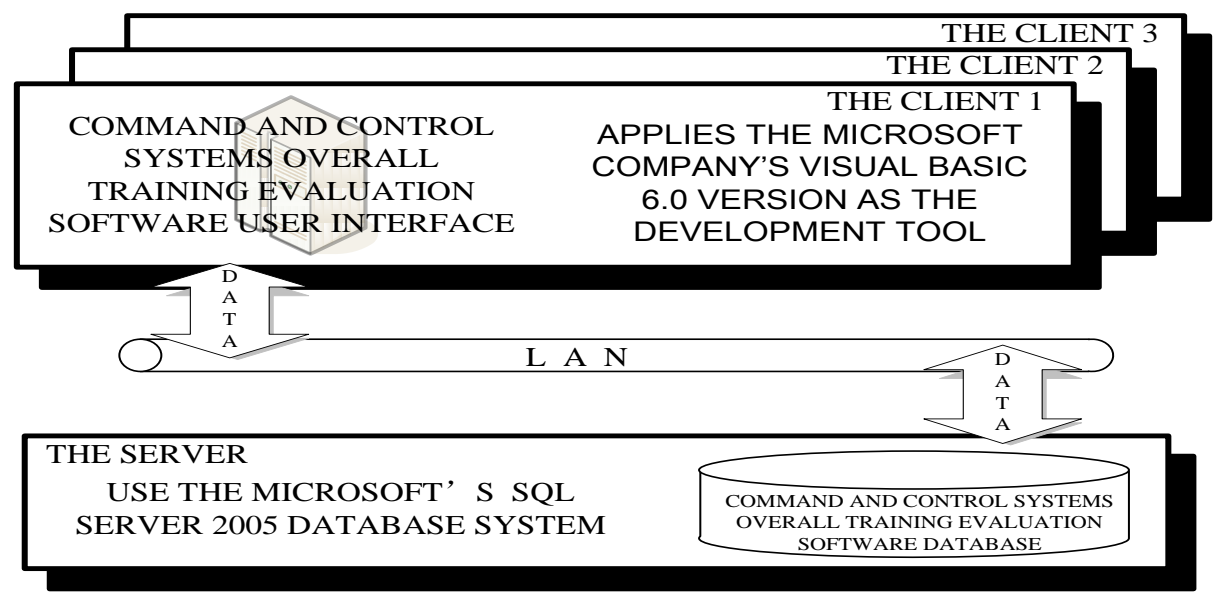

Figure 1. The development environment and the network structure.

\section{B. The Design of Software Function Modules}

The modular design of the command and control system's overall training assessment software directly determines the functional realization of the whole software. [6] According to the specific steps of the command and control system's overall training assessment, we make a precise design of the software function modular by dividing the whole software into seven modules, i.e. User
Information and Access Right Management module, Examination Information Setting Module, Examination Implementation module, Data Processing module, Expert Ability Assessment module, Examination's Result Reading module, and software and database maintenance module. The design of software function modules is shown in Figure 2 .

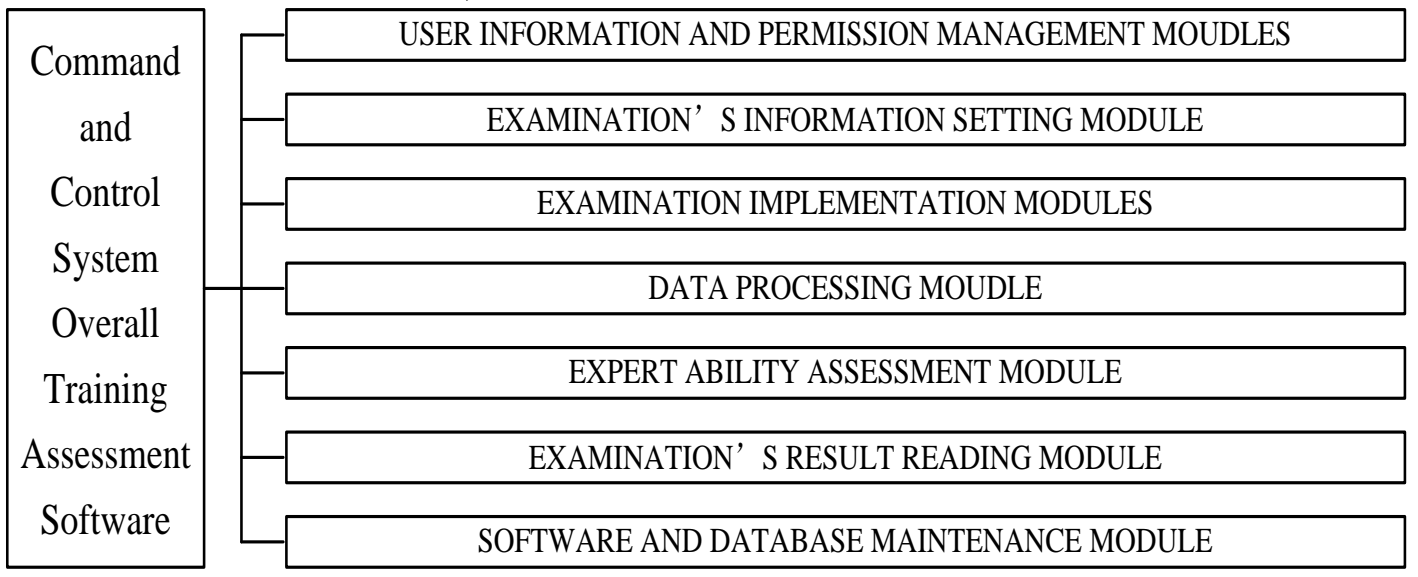

Figure 2. The design of software function module

\section{COMMAND AND CONTROL SYSTEM OVERALL} TRAINING ASSESSMENT MODEL

Being the information processing center, command and decision making center, as well as arms control center of modern surface combatant, the command and control system of new types of destroyer and frigate establishes nine battle stations, which includes the Commanding Officer, the Executive Officer, and seven other roles. [7]To assess the capability of operation teams scientifically, distinguish the different roles, and simplify the operational process, the overall training assessment is constructed on a basis of the characteristics in actual training, having regarding to the Navy Military Training and the Examination Outline. [8] Three aspects are consistently covered by the system, i.e. Intelligence, Command, and General Control. Moreover, the overall training assessment system structure is equipped with clarity, orderliness, completeness and proficiency. The overall training assessment model of the command and control system is shown in Figure 3. 


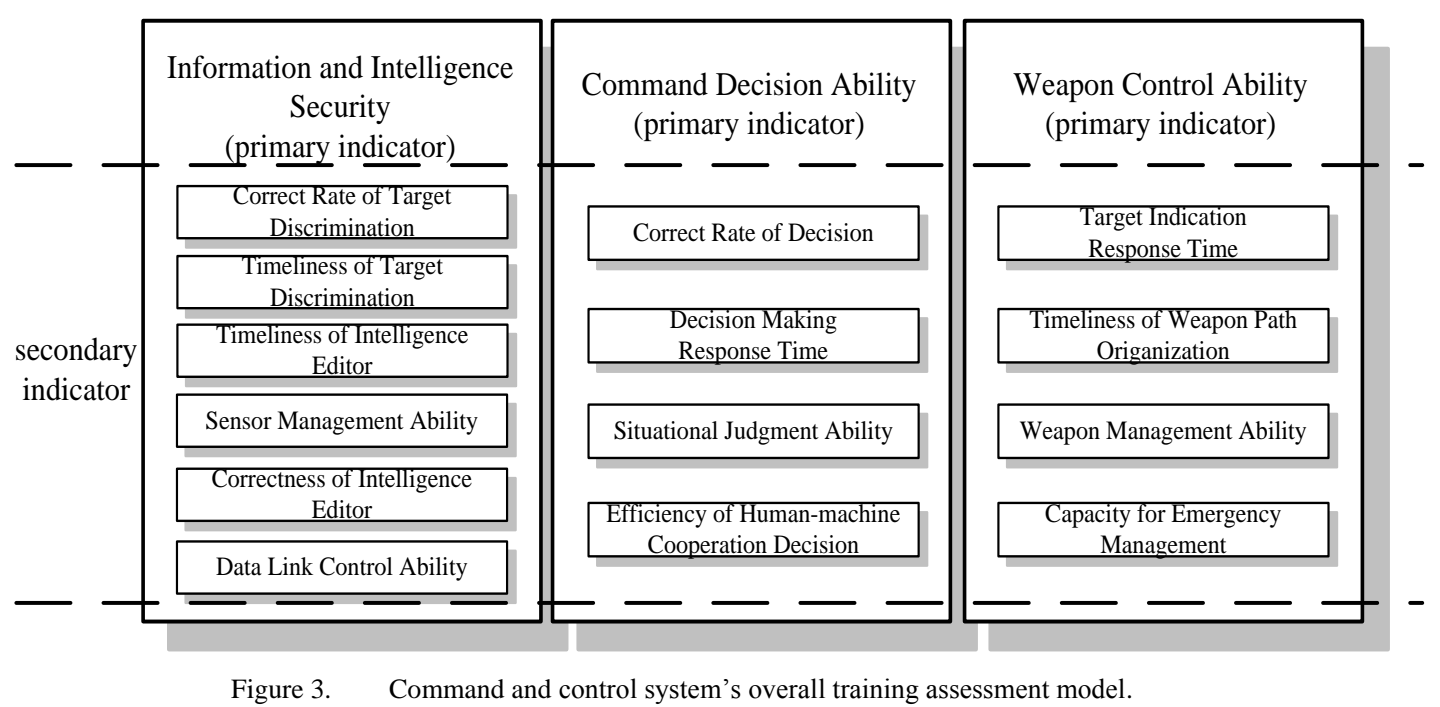

After defining the indicators, the values of the training assessment indicators are determined by the AHP method. This provides the training assessment model for the upcoming design and implementation of the overall training assessment software. [9]

The calculation of the weights of indicators is done by adopting the AHP method. Several primary indicators are defined on a basis of the combat training process, and each primary indicator contains a group of relevant subindicators. Then, we developed a matrix, namely the weight relevance and importance matrix, and created a combined questionnaire for measuring the validity of this matrix. The questionnaire is distributed among experts as well as experienced operators of the command and control system. After the scoring and feedbacks are collected from the questionnaire, the weights of indicators are determined by statistical calculation.

\section{REALIZATION OF ALL SOFTWARE MODULES}

\section{A. User Information and Access Right Management Module}

According to the principle of the practicality and safe reliability of the software, it is necessary for the software to manage the users, distinguish their operating authority, and verify the user's identity when they attempt to log in to the software. The Command and Control System overall training assessment software realizes the management and verification of software user information through "User Information and Access Right Management module", and is able to grant specific rights accordingly. [10]The flow chart of User Information and Access Right Management module is shown in Figure 4.

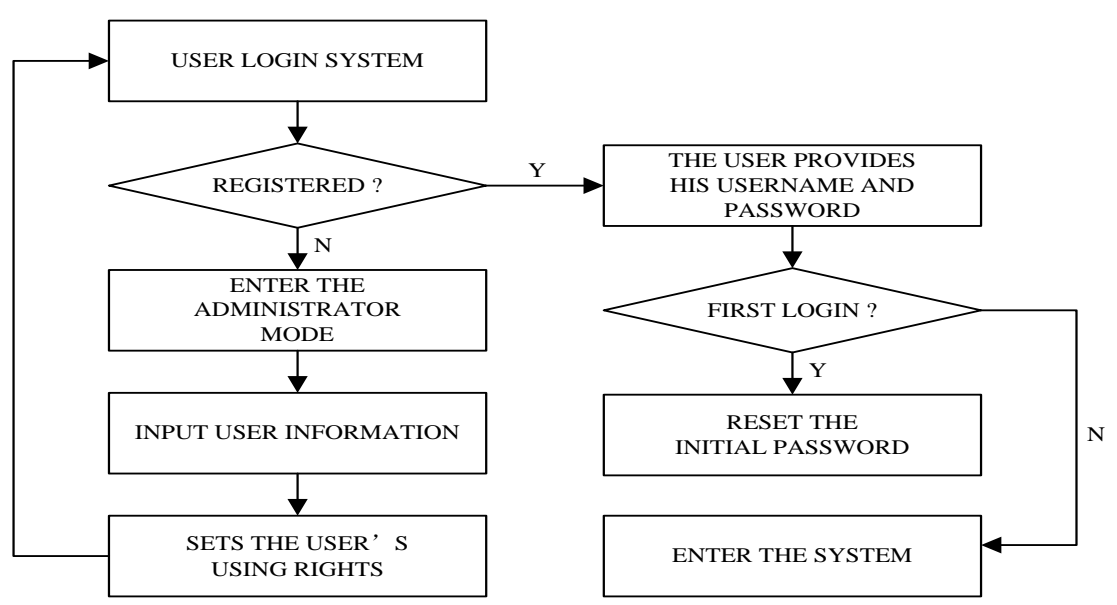

Figure 4. The flow chart of user information and access right management module.

A new software user is required to register through the system administrator. The system administrator enters the personal information of the new user to the user maintenance interface and sets the user rights according to his specific condition. In the process of command and control system overall training assessment, the identity of the examinee varies from one to another, so does the required user rights. In the User Information and Access Right Management module, the exam committee (including experts and recorders) function as executors of the 
examination, and conduct tasks as required by the command and control system examination board. Authority leaders and staffs, as the viewers of the examination's results, check the results and evaluate the training effort. The personnel, who are responsible for managing and maintaining the software will be granted administrative rights not only to maintain the software and data, but also to prepare materials for the examination, i.e. record the exam committee information and examination scores, and set the system variables, etc. After the registration of a new user, the user's personal information will be stored in the database, and the user can have access to the software

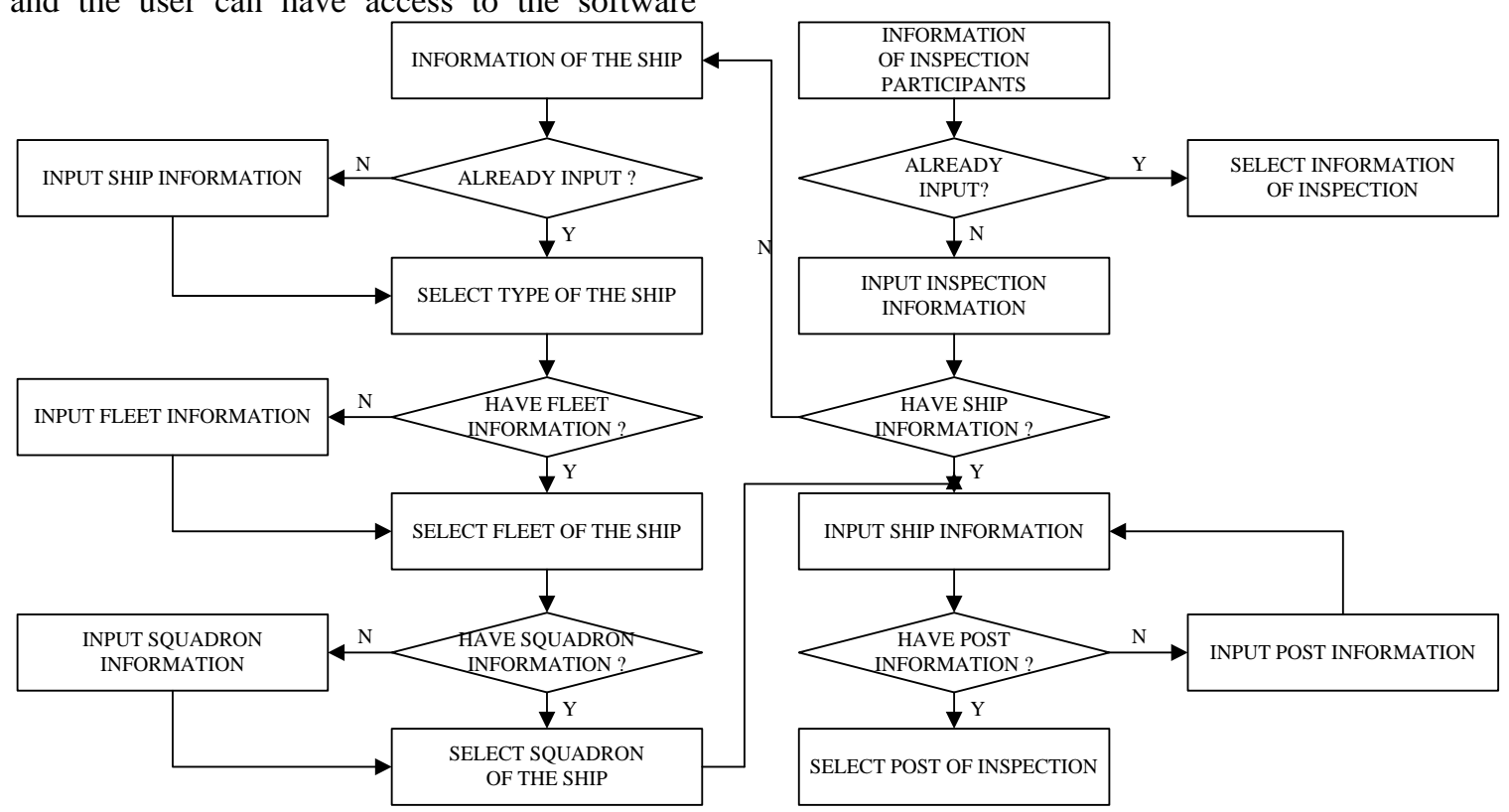

Figure 5. The flow chart of information input module
The personal information of the examinee includes several items, such as name, ID number, belonged ship, battle station, etc. After the examinee's information being stored, the ID number will be regarded as a verifier to confirm his identity in the actual examination. The stored personnel information can be referred to determine the examinee's batch condition during the actual examination. The vessel information includes hull number, ship class, belonged fleet, etc. When an unrecorded value of ship class or unit emerges, it can be added to the database via ship model maintenance interface or unit maintenance interface.

\section{Overall Inspection Implementation Module}

The Overall Training Implementation Evaluation module includes the evaluation details input interface, the reference information input interface, and the overall performance assessment input interface. Regarding those quantitative data in the evaluation, such as operation time and rate of accuracy, the original data will be entered into the specified dialog box as they are; as for qualitative data, such as the assessment of completeness and feasibility of a plan, they require to be scored and commented by experts. through the log in page. For the initial log in, the user is asked to change his password for a security concern.

\section{B. Inspection Information Setting Module}

The Information Input Module for examinees realizes the joint information management of the participants and their work units. Before the examination, the recorder is responsible for importing the examinees' personal information along with the vessel information to the system. Figure 5 displays the flow chart of Information Input Module. module

The initial step during the evaluation is to set the assessment details. This includes information such as batch code, starting time, etc. The following step is to enter the identification code of the examinee (such as ID number) into the examinee selection interface to set up the personnel record of that assessment. If the identification code has not yet existed into the system, the page will jump to the Information Input module, allowing the administrator to create the reference information for that examinee. The final step is to enter the overall training examination batches via the overall operation test interface.

2) The overall implementation evaluation module

In the Overall Training Implementation Evaluation module, regarding those quantitative data in the evaluation, such as operation time and rate of accuracy, the original data will be entered into the specified dialog box as they are. Qualitative data, such as the completeness and feasibility of a plan, require to be scored and commented by experts.

As for the assessment of the "responding time to the instruction", three types of data are being collected: batch code, the start time of instruction, and the end time of 
instruction. After the assessment data is entered by the assessment information recorder into the system, they will be processed by the software, and then the results will be displayed on the record page. If the operator needs to modify or delete a record, he can do so by directly selecting that record. When the operator clicks on the "exit" button, the page can automatically jump to the next assessment input interface and the recorder can efficiently enter the assessment data.

The assessment of "situation judgment" data is collected by both examination scores and expert opinion, and these data will be stored in the database; taking the expert weights into consideration, the scores will be calculated and generated by the software. Meanwhile, the evaluation comments are also stored for future reference. When you the user clicks on the "exit" button, it will jump to the next assessment input interface automatically, the user can enter the other assessment data with higher efficiency.
According to the evaluation criteria, an algorithm is designed and built in the Assessment Data Processing model. The idea is to calculate the operating level of command and control system by a list of inputs such as time and rate of accuracy. In the actual assessment, the weights of assessment indexes often change. To meet the actual requirements, an appraisal index maintenance interface is built to reset the index weights

\section{E. Assessment Results Access Module}

The Assessment Results Access module mainly serves the chief of the institutions and the personnel who perform score review. The functionalities of this module is designed to ensure that the agency of the post can look into the assessment results. Hence, two view modes are created to investigate the achievement: one is the ship hull number view mode, and the other is the assessment projects view mode. Figure 6 displays the flow chart of assessment results access module.

\section{Assessment Data Processing Module}

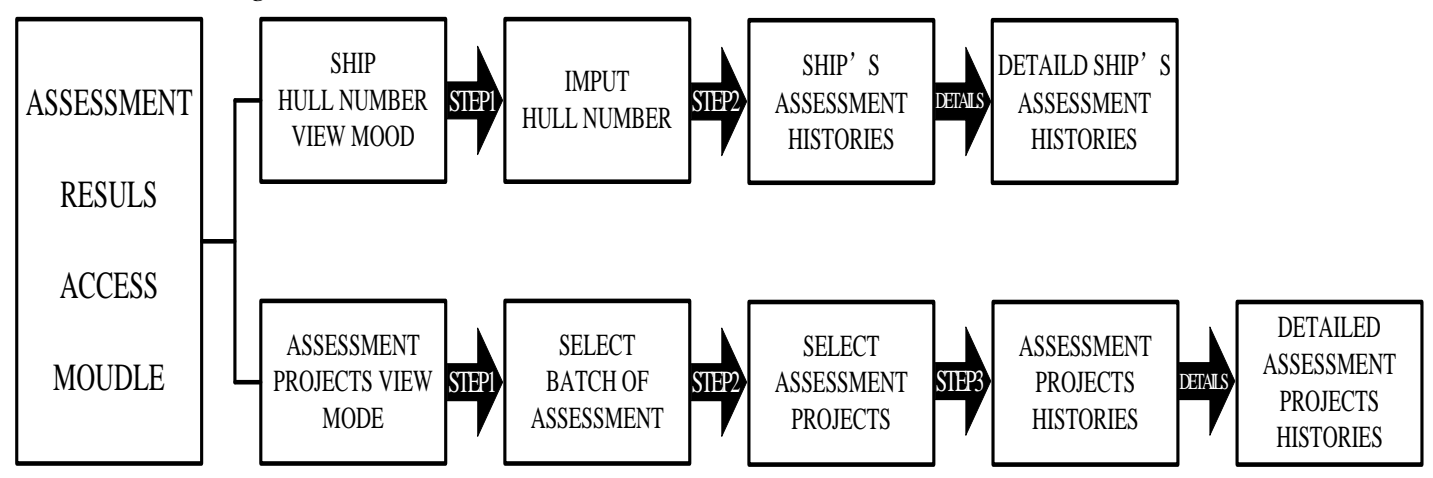

Figure 6. The flow chart of assessment results access module.

1) Ship hull number view mode

In the ship hull number view mode, the system user can enter the ship hull number in his mind, then the user can browse the overview page of the overall training examination of that designated ship, and click on an entry to view the more detailed information of that ship.

2) Assessment projects view mode

In this view mode, at first, the system user should select the assessment items; after that, the user can browse the

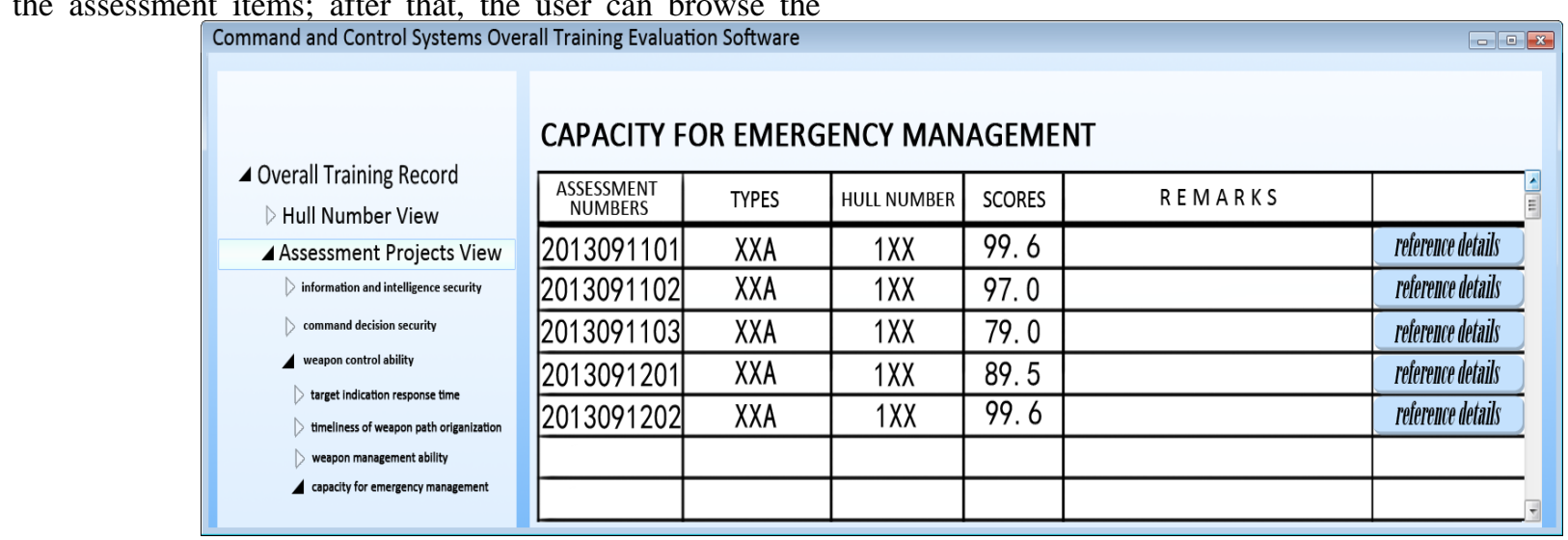

Figure 7. The assessment overview interface. project assessment overview page, which includes assessment numbers, types, ships, ship hull numbers, item scores and other information. The specific interface is shown in Figure 7. By clicking on the reference details, the user can review the specific cases of the batch appraisal of the project overall training assessment, which is convenient for users to detect the problems existed in the personnel training. 


\section{F. Expert Capability Evaluation Module}

The quantitative assessment scores is calculated by an integration of expert opinion. However, it is often the case that the experts who share their opinion do not share the same experience and background. Therefore, he expert opinion should be balanced in the final score calculation. In this module, the quantized expert capability determines the weight of each expert opinion in the final score calculation. The expert evaluation is generated through an pre-designed algorithm, which calculates the weight of the expert capability by a set of inputs, such as the education background, qualification, position, and several other information of the analysed expert. This information are entered and maintained by the system administrator via the recording interface, and stored in the database thereafter.

\section{G. Software and Database Maintenance Module}

This module enables the administrator to maintain the database, minimizes the complexity of work, and meets the users' demands without knowing about the database management. With an interactive interface, the Software and Database Maintenance module realizes functions such as the maintenance of user information, unit information, and warship type information. Those users who are not equipped with professional knowledge of database management or maintenance can also add, delete or modify terms in the database without any difficulty.

\section{LIMITATIONS AND POTENTIAL RESEARCH PATH}

Due to the limitation of the author's theoretical level, the command and control system training assessment proposed in this paper is still mainly aiming at the operating results instead of the operation process, and only solves "where there are questions" instead of "where the questions come from". In the future, the main direction of our research will be the assessment of the command and control system's operation process.

In the command and control system training, the operator often intends to judge his operational effect through the operating results, but not to detect the problems existed in his operation. For most operators, the training time spent to become excellent is way longer than to become qualified. However, the current training assessment software is unable to 1) meet the urgent need of improving the operator's operational level, 2) detect the problems existed in the operator's operational process, and 3) put forward suggestions for improvement. These require the training assessment software to collect the processing amount of the command and control system operation to quantitatively describe the overall operation process of the operator, compare it with the optimal operation model in order to analyse the specific existing problems, and propose suggestions to improve the operation.

In order to achieve the goals mentioned above, on the one hand, the training assessment software should improve the existing operating model. This is because that the current training assessment software is not integrated with the actual one (which includes simulator). Thus, the original examination data has to be manually input to the training assessment software. It has not only lowered the working efficiency of examination assessment, but also run the risk of providing a less detailed assessment of the operator. [11] On the other hand, the training assessment software should consider new approaches of collecting information in the examination process. For example, collect all vital signs of the operator, compare them with the time axis built on a basis of the examination content, and analyse the fluctuation of the physical and mental pressure of the operator during the examination process.

\section{VICONCLUSION}

Approaching the AHP method, this software has optimized the assessment method of the command and control system's overall training examination assessment index and has made the command and control system's overall training assessment more scientific. The principles of software engineering as well as the Object-oriented program design method have been applied. The system is based on the design principles and methods of system standardization. Additionally, the design and development issues of the command and control system's overall training examination assessment are discussed, and finally the software is realized. The proposed system supports the overall training assessment's work requirements, maximizes the value of examination data, and accelerates and enhances the efficiency of the assessment which benefits the senior officers and the superior authority in receiving a timely, accurate, and systematic overview of the training level of the basic army's command and control system. The characteristics of the proposed system (such as ease-of-maintenance, complete functionality, user-friendly interface, high-practicability, and operability, etc.) is in accordance with the current trends of the command and control system's overall training assessment. This system has its strong practical significance in improving the automatic level of the command and control system training assessment and enhancing the scientific nature of the training assessment.

\section{REFERENCES}

[1] Z. Xiu, H. Zhang, Command and Control Systems and Combat Usage, (2014)

[2] S. Zhang, H. ZHANG. New Destroyers and Frigates Command and Control Systems Training Evaluation Software Design and Implementation, 6, 1(2014)

[3] S. Rao, Training and Management of Radar and Information Department, (2006)

[4] L. Zhu, Warship Checking Quality Valuation System Design and Implementation, (2010)

[5] F. Wu, Visual Basic Program Tutorial, (2010)

[6] Z. Guo, J. Chen, SQL Server 2008 Learning Manual, (2011)

[7] Z. Liu, H. Zhang, Database Programming Technology Based on VB and SQL, (2008)

[8] Z. Zhang, J. Xu, Several Problems in the Military Training Evaluation, 144, 5(2012)

[9] Y. He, H. Chen, Evaluation of Joint Training Characteristics of US Army, 99, 5(2010) 
[10] Y. Zhang, Q. Zhu, Grasp the Training Evaluation Improve the Training Efficiency, 22, 3(2010)
[11]L. Zhu, Warship Checking Quality Valuation System Design and Implementation, (2010)

[12] K. Ryan, R. Ronald, Database Design, 133, (2007) 